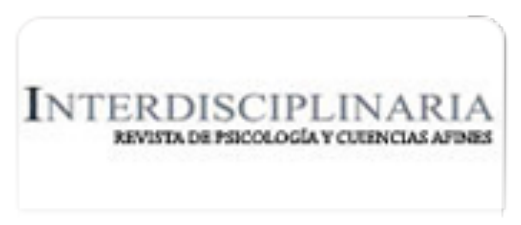

Interdisciplinaria

ISSN: 0325-8203

ISSN: 1668-7027

interdisciplinaria@fibercorp.com.ar

Centro Interamericano de Investigaciones Psicológicas y

Ciencias Afines

Argentina

Ramos-Galarza, Carlos

Construcción del déficit de atención / hiperactividad en la prensa escrita ecuatoriana (2007־2015)

Interdisciplinaria, vol. 35, núm. 2, 2018, Julio-, pp. 363-380

Centro Interamericano de Investigaciones Psicológicas y Ciencias Afines

Argentina

Disponible en: https://www.redalyc.org/articulo.oa?id=18058785008

Cómo citar el artículo

Número completo

Más información del artículo

Página de la revista en redalyc.org

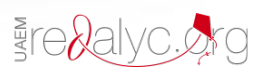

Sistema de Información Científica Redalyc

Red de Revistas Científicas de América Latina y el Caribe, España y Portugal Proyecto académico sin fines de lucro, desarrollado bajo la iniciativa de acceso abierto 


\title{
Construcción del déficit de atención / hiperactividad en la prensa escrita ecuatoriana (2007-2015)
}

\section{Construction of attention deficit / hyperactivity disorder in Ecuadorian written media (2007-2015)}

\author{
Carlos Ramos-Galarza* \\ *Psicólogo. Máster en Neuropsicología Clínica. PhD en Psicología. Profesor Principal Titular de la Pontificia \\ Universidad Católica del Ecuador. Investigador del Centro de Mecatrónica y Sistemas Interactivos de la \\ Universidad Tecnológica Indoamérica de Ecuador. E-mail: ps_carlosramos@hotmail.com
}

Departamento de Psicología. Universidad Internacional SEK del Ecuador. Quito, Ecuador.

\begin{abstract}
Resumen
El trastorno por déficit de atención con hiperactividad (TDAH) es una alteración del neurodesarrollo que afecta al comportamiento y cognición de niños/as, adolescentes y adultos. En torno a su línea de investigación, actualmente no se cuenta con una teoría contundente que permita comprenderlo en su totalidad, por lo que todavía se encuentra en desarrollo su fundamentación teórica, determinación de factores causales, eficacia de tratamientos y demás aspectos que engloban al TDAH. En tal contexto, en el presente artículo se propone una investigación que analiza su cobertura otorgada en medios de comunicación, específicamente en la prensa escrita de Ecuador entre 2007 y 2015. La metodología utilizada se basa en dos fases: una primera cualitativa, en donde se hizo un análisis de contenido de treinta y nueve reportajes sobre el trastorno. En la segunda fase se analizaron las frecuencias, porcentajes y coeficiente de confiabilidad de Kappa de la medición realizada. En los resultados encontrados de la fase cualitativa, se propone un paradigma codificado constituido por once categorías que construyen el conocimiento del TDAH en la prensa escrita de Ecuador, donde se resaltan aspectos como las alteraciones familiares, educativas y sociales que genera este trastorno en las personas que lo presentan, mitos sobre sus posibles causas, creencias en torno a la eficacia y efectos no deseados
\end{abstract}

del fármaco como medio de tratamiento, factores de riesgo, protección y otras categorías que se proyectan de los contenidos de los reportajes analizados. En la valoración cuantitativa, se encontró que la evaluación interjueces es de $k=.75$ que se interpreta como altamente concordante a la medición realizada. Finalmente, se discuten y analizan los resultados en torno a investigaciones previas y la teoría del TDAH.

Palabras clave: Déficit de atención; Hiperactividad; Impulsividad; Prensa escrita de Ecuador; TDAH.

\begin{abstract}
Attention deficit hyperactivity disorder (ADHD) is an alteration of neurodevelopment that affects the behavior and cognition of children, adolescents and adults. ADHD encompasses three clinical features: excessive motor activity, impaired impulse control, and clinically significant alterations in the ability to focus attention to internal and external stimuli. Regarding its research line, there is currently no strong theory to understand it in its totality, so that it is still developing its theoretical foundation, since there are several proposals that explain the development of this disorder from genetic, the influence of diet, social context, family, and other aspects. Another topic still under construction of
\end{abstract}


ADHD is the efficacy of psychological and neurological treatments, alternative therapies and other proposals of treatment that are used for ADHD. In this context, and contributing to the theoretical construction of ADHD, one of the aspects that have not yet been investigated is how this disorder is constructed in the different contexts in which information about its characteristics is handled. One of them is in the media where it say several statements about ADHD that influence the understanding of this disorder by parents, relatives, teachers and the same people who present it. This article proposes an investigation that analyzes the coverage given to ADHD in the media, specifically in the written press of Ecuador between 2007 and 2015. The methodology was based on a mixed research design of two phases. In the first phase, a qualitative procedure of content analysis of the narratives, contained in thirty-nine reports of the Ecuadorian written press about the disorder was used. In the second phase, through a descriptive quantitative research design, we analyzed the frequencies and percentages of each of the reports and their quantitative contribution in the conformation of the categories found in the qualitative phase. In addition we calculated the Kappa reliability coefficient of the measurement performed by means of the procedure inter judges. In the results of qualitative phase, we propose a codified paradigm constituted by eleven categories that construct the knowledge of ADHD in the written press of Ecuador, where they emphasize aspects such as the family, educational and social alterations that generates this disorder in the people that present it, myths about its possible causes, beliefs about the efficacy and unwanted effects of the drug as a means of treatment, risk and protection factors, and other categories that are projected from the contents of the analyzed articles. In the quantitative evaluation the numerical values that constructed the eleven categories of the qualitative phase are exposed through frequencies and percentages. In the analysis of Cohen's Kappa coefficient, we found that the inter-evaluation is $k=$ .75 , which is interpreted as highly concordant to the measurement performed. Finally, the data reported in the qualitative and quantitative phases are discussed around the theories about ADHD reported in the main diagnostic manuals and with investigations that affirm the information of the reports that informs the real aspects of the disorder, which contribute to a responsible management of the knowledge about the ADHD and contribute in the understanding and help of children, adolescents and adults that have this disorder. Instead, some myths about ADHD are discussed in the reports and contrasted with formal theory and research on the subject, so that myth-based stories are critically received, as they may affect the beliefs of people living around people with ADHD.

Keywords: Attention deficit disorder; Hyperactivity; Impulsiveness; Ecuadorian written press; ADHD.

\section{Introducción}

La Asociación Americana de Psiquiatría (APA, 2013) describe que el trastorno por déficit de atención con hiperactividad (en adelante TDAH) es un trastorno del neurodesarrollo que se caracteriza por la presencia de un patrón elevado de actividad motora, impulsividad y dificultades de atención. En diversas investigaciones se ha reportado que el TDAH es un cuadro altamente recurrente y estaría presente aproximadamente entre el 3\% y el10\% de la población (De la Barra, Vicente, Saldivia, \& Melipillan, 2013; Thomas, Sanders, Doust, Beller, \& Glasziou, 2015; Vélez-Van-Meerbeke, Zamora, Guzmán, López, \& TaleroGutierrez, 2013).

Uno de los aspectos controversiales en la comprensión del TDAH radica en que, actualmente, no se cuenta con una sólida teoría que permita comprender de forma definitiva los factores causales del cuadro, procedimientos adecuados de intervención o modelos explicativos que permitan controlar o predecir este trastorno (Nigg, 2012). Es abundante la evidencia empírica reportada en torno al TDAH. Por ejemplo, se han realizado diversas investigaciones internacionales sobre el funcionamiento cognitivo en niños/as y adolescentes con el 
trastorno (Winther, Egeland, Norman, Tore, \& Øie, 2014), la evaluación del desempeño atencional en el medio escolar (Monteoliva, Ison, \& Pattini, 2014), análisis de sus características neurofisiológicas (Roca, Presentación-Herrero, Miranda-Casas, Mulas, \& Ortiz-Sánchez, 2014), desempeño académico y de comportamiento en el medio educativo (Anderson \& Reidy, 2012), eficacia al intervenir en el cuadro mediante el tratamiento farmacológico (Biederman et al., 2002), innovaciones tecnológicas para mejorar su aprendizaje (Raposo-Rivas \& Salgado-Rodríguez, 2015), análisis del papel de los neurotransmisores como factor de riesgo en el trastorno (Naaijen et al., 2017), alteraciones de las funciones ejecutivas en niños/as con TDAH (Graham, 2017 ), su relación con el uso excesivo de juegos electrónicos (Benjamin, Peter, \& Lynne, 2017), experimentos de entrenamiento cognitivo y conductual (Miranda \& Presentación, 2000), entre otros.

A pesar de la cuantiosa evidencia empírica reportada en torno al TDAH, todavía existen áreas que no han sido exploradas y paradigmas científicos desde los cuales no se ha abordado este trastorno. Por ejemplo, no existe evidencia, desde un paradigma constructivista, de la percepción de la realidad construida alrededor del TDAH (Ramos, 2015), la subjetividad edificada por parte de pacientes y familiares con el trastorno, los mitos sobre su tratamiento o la influencia de los medios de comunicación en la información o desinformación de lo que engloba el TDAH.

En el contexto ecuatoriano, delimitación contextual del presente estudio, existe un desarrollo incipiente de la línea de investigación del TDAH. Tras la revisión del estado del arte en las principales bases de datos de investigaciones (Latindex, Scopus y Web of Science), no se han encontrado estudios que hayan respondido a la pregunta: cómo se construye al TDAH desde las diferentes dimensiones que componen a la sociedad, como por ejemplo, desde la influencia que ejercen los medios de comunicación en la construcción de la realidad y los imaginarios de las personas.
Dentro de dicha descripción, es de vital importancia contribuir con el desarrollo de la línea de investigación del TDAH en Ecuador mediante la realización de estudios que aporten evidencia empírica sobre el manejo terapéutico del cuadro en el país, sus procesos diagnósticos, controversias, construcciones discursivas desde las esferas expertas y los diferentes medios de expresión social, como son los medios de comunicación, considerados como los voceros de la comunidad y demás campos de interés todavía no analizados en el país.

En el Ecuador, los medios de comunicación escrita, de manera específica los periódicos de circulación nacional, han brindado varios espacios para la publicación de noticias relacionadas con el TDAH, en donde se han relatado, sobre todo, temas relacionados con sus causas y tratamiento. Dentro de esta descripción se inserta el objetivo principal del presente estudio, que será analizar qué significados se han construido en torno a este trastorno desde los medios de comunicación escrita de circulación en Ecuador y contrastarlos con la teoría y hallazgos de investigaciones empíricas.

La realización de este estudio es un importante aporte dentro de la línea de investigación del TDAH, ya que es vital identificar la información que se maneja en la sociedad ecuatoriana en relación a este trastorno, teniendo como marco de referencia el interaccionismo simbólico de los medios de comunicación, lectores y las construcciones imaginarias que se generan sobre el trastorno en los pacientes, familiares, profesionales y en todos quienes viven el TDAH.

Además, es importante mencionar al lector que este análisis se realiza desde una postura psicológica, que permita aportar al clínico que brinda servicio a seres humanos con TDAH, la comprensión de los constructos que emergen sobre este trastorno en los reportajes escritos en Ecuador, ya que esta sistematización permitirá un acercamiento a la interpretación de mitos y verdades que existen en torno a este trastorno 
neuropsicológico en los imaginarios sociales del país.

\section{Método}

\section{Materiales}

Para llevar a cabo la investigación se procedió a revisar las bases de datos de todos los medios de comunicación escrita de Ecuador, donde se encontraron treinta y nueve reportajes sobre el TDAH (publicados desde 2007 hasta 2015) en los diarios El Comercio, El Universo, El Telégrafo, La Hora, El Mercurio, El Diario y Últimas Noticias (en adelante, EC, EU, ET, LH, EM, ED y UN, respectivamente).

\section{Diseño de investigación}

El estudio se basó en un diseño de investigación mixto de dos etapas denominado diseño mixto secuencial confirmatorio. Según Hernández, Fernández y Baptista (2010), este tipo de investigaciones se conforman por dos etapas, en las que se utilizan los diseños cualitativos y cuantitativos para lograr los objetivos de una investigación. En tal sentido, en la primera fase del estudio, se utilizó un diseño cualitativo de teoría fundamentada (Strauss \& Corbin, 2002), y en la segunda fase, se utilizó un diseño de investigación cuantitativo descriptivo (Hueso \& Cascant, 2012).

\section{Procedimiento}

Posteriormente a la revisión de los diarios, se organizaron los reportajes según una posible temática, englobando aspectos del TDAH como: tratamiento, causas, factores relacionados, historias de casos, creencias sobre la medicación, postura de padres y madres ante el tratamiento, entre otros tópicos que aportaron a la construcción de la teoría fundamentada de la presente investigación. Posteriormente, se aplicaron las técnicas de análisis de datos cualitativos y cuantitativos. El análisis cua- litativo se lo realizó bajo las directrices del programa Atlas.ti, mientras que en el análisis estadístico se utilizó el programa SPSS versión 20 (IBM, 2011).

\section{Análisis de datos}

Para la fase cualitativa se utilizó el procedimiento de análisis de contenido de los reportajes encontrados. En la segunda fase, destinada a realizar el análisis cuantitativo, se utilizó un procedimiento estadístico descriptivo, en el que se aplicaron técnicas de análisis de frecuencias y porcentajes. Mediante este procedimiento cuantitativo se describió la cantidad de reportajes y el porcentaje de aporte de cada artículo en la construcción de las categorías identificadas en la fase cualitativa. Además, para confirmar la confiabilidad de la medición realizada, se calculó el coeficiente Kappa de Cohen mediante la valoración interjueces.

\section{Resultados}

\section{Fase cualitativa}

Luego de realizado el proceso de codificación abierta del contenido lingüístico de los reportajes encontrados en los medios de comunicación escrita del Ecuador, se pudo interpretar la construcción del TDAH desde las categorías: origen del TDAH; el TDAH produce alteraciones familiares, educativas y sociales; el medicamento que lucha contra el TDAH; el concepto del TDAH en la prensa; efectos del fármaco en el TDAH; factores protectores en el TDAH, y la frecuencia del TDAH y su diagnóstico. A continuación, se describe el contenido de cada una de las categorías.

\section{Origen del TDAH.}

En esta categoría se engloban conceptos que describen factores relacionados con las causas del TDAH manifestadas en los diferentes diarios analizados. En primer lugar, emergió la concepción de que el TDAH es 
producto de un déficit del sistema nervioso. EC (2015d) y LH (2012c) exponen que este trastorno es consecuencia de un problema cerebral a un nivel de las estructuras orgánicas implicadas en la regulación del comportamiento. Según ED (2014b), una de las principales alteraciones a nivel cerebral en el TDAH, engloba una disfunción a nivel del neurotransmisor dopamina -que tiene una importante incidencia en el control de la conducta del ser humano- y que probablemente generaría la sintomatología que presentan niños, adolescentes y adultos con el trastorno.

El segundo factor que emerge como una variable causal del TDAH, es el uso de dispositivos electrónicos. Según EC (2012c) el uso que dan los niños a dispositivos como tabletas digitales o celulares, podrían desencadenar el TDAH. En la misma línea, EC (2012b) afirma que la exposición de un gestante, durante su fase de embarazo, a la radiación producida por los dispositivos electrónicos como celulares, dispositivos de localización, WIFI y demás artefactos que se comunican por vía inalámbrica, podrían generar una influencia negativa en el proceso de conformación de las estructuras cerebrales del futuro niño, ya que si bien las frecuencias eléctricas emitidas no son percibidas por el ojo humano, éstas serían capaces de llegar a influir de manera negativa en la estructuración celular del sistema nervioso.

El tercer factor que emerge en las narrativas de los reportajes expone diversas situaciones contextuales y personales que podrían aumentar el riesgo de generar un TDAH. EC (2013a) propone que cuando un niño posee varios cuadros psicopatológicos, como por ejemplo autismo, ansiedad, negativismo, agresividad y problemas emocionales, tendría más probabilidades de ser diagnosticado con TDAH. EC (2011b) afirma que existen situaciones contextuales que también aumentan el riesgo en un niño de presentar TDAH, como puede ser, el vivir junto a un fumador. ET (2014) propone como factor de riesgo, la ingesta por parte de la madre, de fármacos antidepresivos du- rante el primer trimestre del embarazo. LH (2012b) indica que los niños pequeños que roncan o sufren problemas respiratorios tienen más probabilidad de presentar sintomatología del TDAH, a diferencia de los que duermen sin hacer ruido alguno o no presentan problemas de este tipo. Según EC (2010b, 2012a, 2015b) y LH (2012c, 2015) la herencia genética, problemas durante el embarazo o en el nacimiento también podrían ser factores causantes del TDAH.

Además de los factores identificados previamente en los contenidos lingüísticos de los reportajes, surgen dos posturas. Por un lado, una propuesta para dejar de lado ciertas creencias asociadas como factores causales del TDAH, y por otro, una propuesta de afirmar ciertos mitos en torno al trastorno.

En tal sentido, EU (2011c) y LH (2012a) proponen una desmitificación de varios factores que no producirían TDAH, como por ejemplo el consumo de azúcar, ver demasiada televisión, mal control del niño por parte de sus padres y madres, factores ambientales y sociales como la pobreza, el caos familiar o el consumo excesivo de aditivos alimentarios; que constituyen factores que no tendrían una relación causal, ya que el TDAH tendría una fuerte relación con factores de tipo neurobiológicos.

En la segunda perspectiva, que describe ciertas creencias en torno a la comprensión de los factores etiológicos del TDAH, EU (2010) menciona que este trastorno podría ser un producto de una mala educación y un consumo desmesurado de azúcar, en donde, la ingesta excesiva de dulces y colorantes artificiales, generaría déficits de dopamina, que sería la causa principal de las dificultades en la regulación del comportamiento característico del TDAH (ET, 2013b; EC, 2013b).

Como se ha podido identificar hasta este punto, existen varios factores que los diarios atribuyen como causalidad del TDAH, en donde se observa que la discusión sobre el tema no está concluida, de manera que, y como lo describen LH (2015) y EU (2011c), la actualidad no existe un conocimiento ab- 
soluto de las causas del TDAH, sino un acercamiento en el entendimiento de los factores etiológicos del trastorno, ya que éste todavía se encuentra en desarrollo y comprensión por la comunidad científica, lo cual invita a que la lectura que se realice de todas las posibles explicaciones del TDAH, se lo haga desde un paradigma crítico que tenga en cuenta que en este fenómeno todavía no está dicha la última palabra, debido a que no hay conclusiones definitivas en sus investigaciones y/o propuestas teóricas.

\section{EI TDAH produce alteraciones fami- liares, educativas y sociales.}

En la narrativa de esta categoría los niños con TDAH son descritos como individuos agresivos, incapaces de mantener una adecuada relación interpersonal y limitados para establecer un proceso de juego normal, además de ser culpables de la mala dinámica familiar (EC, 2015b, EC, 2010b). Según EU (2010) los niños con TDAH presentan dificultades en el medio educativo, siendo el bajo rendimiento académico y las dificultades de interrelación que se presentan con los compañeros sus mayores problemáticas (EU, 2009). En la misma línea, EC (2015b) asevera que los niños con TDAH son incapaces de controlar su comportamiento y presentan graves dificultades para concentrarse, por lo que no pueden ser integrados dentro del sistema educativo normal (EU, 2012a; LH, 2015).

Las situaciones descritas previamente no son condiciones aisladas, sino que, según el diario UN (2013), evolucionan desde temprana edad; por ejemplo, existen casos que pueden iniciarse desde la etapa preescolar, en donde se puede observar que un niño con dificultades en la articulación de las palabras podría desencadenar un TDAH en la etapa posterior del desarrollo, lo cual constituye una primera llamada de atención a tomar en cuenta en el desarrollo infantil, ya que en etapas posteriores del desarrollo podría desencadenar en un cuadro de mayor complejidad y generar, como se afirmó previamente, alteraciones familiares, educativas y sociales.

\section{El medicamento que lucha contra el TDAH.}

En la narrativa que emerge en esta categoría se sugiere que el fármaco es la principal estrategia que beneficia al comportamiento de los niños con TDAH, manteniendo estable su desempeño ya que este procedimiento le permite controlarse, incluso si lo dejara de consumir (EC, 2015b; EU, 2011a; EU, 2012a). Para LH (2012c) el medicamento ayuda al niño con TDAH a concentrarse, mejorar en el aprendizaje y estar tranquilo, lo cual implica mejorar el comportamiento en el medio educativo al ayudarlo a focalizar la atención en una sola cosa a la vez. De manera que le permite corregir sus problemas de aprendizaje y mantener relaciones sociales estables $(\mathrm{LH}$, 2015).

Según EC (2010a, 2015a) y EU (2010) cuando un niño con TDAH se beneficia del tratamiento farmacológico se reducen las posibilidades de que pueda sufrir accidentes o una muerte prematura en un futuro, por lo que es importante detectarlo en forma temprana para iniciar su intervención oportunamente. La única problemática del uso del fármaco en el TDAH, según LH (2012c) y EU (2012a) radica en que los niños que empiezan a recibir este tipo de intervención deben experimentar con varias dosis y tipos de fármacos hasta encontrar el ideal para su tratamiento, siendo uno de los factores que más preocupa a los padres, ya que en ocasiones se generan efectos no deseados en la etapa de prueba de la dosis del fármaco.

El diario LH (2012c) afirma que la terapia farmacológica en combinación con el tratamiento psicológico de tipo conductual, benefician en mayor medida al TDAH, a diferencia de la aplicación de sólo un tipo de tratamiento, ya que con ambas técnicas se estaría actuando en la condición neurobiológica y comportamental que engloba a este trastorno.

A pesar de la descripción realizada en cuanto al tratamiento propuesto para el TDAH, en EU (2012a) se menciona que el niño con TDAH es un ser humano que demanda más alternativas de tratamiento, un 
individuo que pide a gritos ser más escuchado por sus terapeutas, un individuo que no entiende su enfermedad, en general, un niño que anhela volver a ser normal.

\section{Efectos del fármaco en el TDAH.}

En este concepto emergieron dos categorías. En primer lugar, información relacionada con los efectos no deseados que genera el fármaco en el tratamiento del TDAH y, por otro, una tendencia a desmentir posibles riesgos de muerte originados en el consumo del medicamento.

Según EU (2009) los niños con TDAH que reciben medicamento corren el riesgo de presentar efectos no deseados como psicosis o manía, e incluso existen infantes que mencionan haber alucinado ver monstruos, gusanos o bichos que trepan sobre su cuerpo después de haber iniciado el consumo del fármaco. En otros artículos, se expone que los niños con TDAH que reciben el fármaco empiezan a presentar un comportamiento parecido al de un zombi (EU, 2012a) y que el medicamento puede generar efectos no deseados como dolores de estómago y problemas del sueño $(\mathrm{LH}$, 2012c).

La segunda categoría tiene relación con desmentir los efectos no deseados generados por el consumo del fármaco. En tal sentido, EC (2011a), EU (2011a, 2011b) y EM (2011) indican que el uso de medicamentos para tratar el TDAH no generaría una muerte súbita, infarto al miocardio o algún accidente cerebro vascular, que es una información que ha generado preocupación en los padres de pacientes que reciben este tipo de tratamiento. Finalmente, realizando una especie de advertencia de lo que pudiera suceder en el futuro si no se consume el medicamento para tratar el TDAH, en EC (2015b) se afirma que cuando un niño con TDAH no recibe tratamiento para controlar su cuadro, es probable que en el futuro pueda presentar fracaso escolar y social.

\section{Concepto del TDAH construido por la prensa: características del cuadro.}

Según EM (2013) el TDAH es una situación real que afecta a un importante porcentaje de la sociedad, en donde el niño con el trastorno es un individuo que no puede controlarse, que no se encuentra dentro de los estándares del comportamiento establecido de acuerdo a los parámetros cognoscitivos y de comportamiento social de su edad, por lo que debería beneficiarse de un proceso terapéutico para ser integrado a la sociedad (EC, 2015b).

EU (2010) describe que el TDAH es un trastorno que engloba un comportamiento caracterizado por la presencia de una excesiva actividad motora, impulsividad y desatención. Para LH (2012c) se trata de un niño que está en constante movimiento, habla incesantemente o tiene problemas para concentrarse en las actividades que debe realizar. Según LH (2015) este trastorno aparece a la vista cuando el niño se encuentra entre los seis o siete años de edad, cuando ingresa a la escuela y se hacen evidentes sus problemas en la realización de sus actividades pedagógicas, en las cuales debe concentrarse y mantener un comportamiento autorregulado.

\section{Factores protectores en el TDAH.}

Un factor protector, a diferencia del factor de riesgo, evita o disminuye la probabilidad de la aparición de una posible condición psicopatológica (Cova, 2004). En tal sentido, ET (2013a) y ED (2013) describen que los bebés que se benefician de lactancia materna presentan menos probabilidad de desarrollar el TDAH, en relación con los infantes alimentados con fórmula láctica química o que carecen de este tipo de alimentación.

En EC (2015b) se testifica que una institución educativa en la cual se realicen los cambios en el proceso de enseñanza y aprendizaje del niño con TDAH es un factor que beneficia su comportamiento y rendimiento educativo, a diferencia de las ins- 
tituciones educativas donde no se adapta el sistema al niño con TDAH, donde por lo general, éste termina fracasando.

Además, en EU (2012a) se propone que la familia de un niño con TDAH debería recibir educación sobre el tema, lo cual permitiría a los familiares del menor poder comprender el cuadro y por ende, brindarle una atención necesaria tomando las debidas decisiones para acudir a la terapia y realizando los cambios necesarios dentro del contexto familiar, en pro del comportamiento y cognición del niño.

\section{Frecuencia del TDAH y su diagnóstico.}

EC (2010b), EU (2010) y LH (2007) exponen que el TDAH es el motivo de consulta más frecuente en la atención infantil, ya que en los departamentos de Psiquiatría de Ecuador, el motivo de consulta más común es la hiperactividad y el déficit de atención. En cuanto a su prevalencia, para LH (2012c) el 9.5\% de los infantes tendrían TDAH y para el diario UN (2013) el 30\% de la población presentaría el trastorno.

En cuanto al proceso diagnóstico del TDAH, EC (2010a, 2015b) afirma que no se debe mencionar que un niño tiene el trastorno si no ha existido un diagnóstico emitido por un especialista. Además, este proceso debería seguir el orden de primero acudir a un neurólogo, posteriormente a un psiquiatra y luego a un psicólogo. Dentro de este contexto, LH (2015) sugiere que antes de medicar a un niño se debe analizar si realmente presenta el trastorno, puesto que existen casos en los cuales ni siquiera se ha confirmado la presencia de la sintomatología del TDAH y ya se inicia con la suministración del fármaco.

Como dato curioso en este campo del diagnóstico del TDAH, según los diarios el EC (2010a) y EM (2011) el TDAH también ha estado presente en grandes hombres dentro de la historia de la humanidad, como Einstein, Galileo Galilei, Edison, Mozart, Walt Disney, Ford, entre otros; además de algunos no tan famosos por aportes a la humanidad y que justifican su comporta- miento disruptivo por la presencia del trastorno.

Finalmente, en EC (2015a) se expone que es importante que el TDAH sea diagnosticado tempranamente en el género femenino, ya que produciría mayores riesgos de presentar una muerte prematura, debido a los mayores índices de impulsividad presente en pacientes mujeres con TDAH.

\section{Paradigma codificado.}

En la Figura 1 se presenta un esquema gráfico en el cual se hace una propuesta de organización de las relaciones de las categorías de la teoría que emergió en la presente investigación.

En el paradigma codificado del fenómeno en estudio se pueden identificar las siguientes relaciones. Mediante el análisis de codificación axial, se identificó a C1 (la letra $\mathrm{C}$ significa categoría) como la categoría principal o central del trastorno, ya que es el aspecto más mencionado sobre el TDAH en los medios de comunicación escrita de Ecuador. C3 es la conceptualización que se realiza sobre el TDAH, que es construida por los medios de comunicación ecuatorianos que han publicado reportajes en torno a este trastorno que aqueja a un sector importante de la sociedad.

Las categorías C4, C7 y C9 son los factores de causalidad que podrían producir el TDAH en un ser humano. Sin embargo, si entre dicha relación causal está presente la categoría $\mathrm{C} 10$, la probabilidad de que se genere el trastorno disminuye considerablemente, ya que los factores protectores intervendrían en dicha relación de causalidad, atenuando el impacto de los factores causales en el TDAH.

La categoría $\mathrm{C} 2$ es el medio de tratamiento mayormente descrito como beneficio en el TDAH, que a la vez que resuelve el problema, también podría generar ciertos efectos no esperados en el paciente que recibe el tratamiento. En tal sentido, las categorías $\mathrm{C} 11$ y $\mathrm{C} 5$, se desprenden del uso del medicamento en el TDAH, puesto que buscan describir los efectos no deseados en los 
pacientes que reciben el fármaco y, por otro lado, desmentir los riesgos que puede causar el uso de este procedimiento.

Finalmente, las categorías C6 y $\mathrm{C} 8$ se desprenden de la construcción que se ha realizado en torno al TDAH, ya que informan la frecuencia en la cual este trastorno se presenta en el medio ecuatoriano y, por otro lado, la importancia del proceso diagnóstico que se debe seguir en este trastorno.

\section{Fase cuantitativa.}

En la Figura 2, mediante un procedimiento basado en estadística descriptiva de frecuencias y porcentajes, se presenta la cantidad de artículos y reportajes que construyeron cada una de las categorías. Por ejemplo, en el tema concerniente a la producción de alteraciones familiares, educativas y sociales en el TDAH, se identificó como la categoría con mayor cobertura en la prensa escrita de Ecuador. Posteriormente se pueden identificar, en orden descendente, el resto de categorías que fueron emergiendo en el discurso sobre el TDAH descrito en los artículos analizados.

En la Tabla 1 se indican los porcentajes con los que cada diario participó en la construcción de las diferentes categorías presentadas en la teoría fundamentada descrita en la fase cualitativa. Con la finalidad de identificar la confiabilidad de la medición realizada, se aplicó el coeficiente Kappa de Cohen de evaluación de la concordancia interjueces, donde se obtuvo un resultado de $k=.75$, que se interpreta como sustancialmente concordante.

\section{Discusión y conclusiones}

El presente artículo se planteó como objetivo identificar los significados que los medios de comunicación escrita del Ecuador construyen en torno al TDAH. Para esto se analizaron todos los reportajes sobre este trastorno que han sido publicados en los diarios: EC, EU, ET, LH, EM, ED y UN.

El principal significado construido en torno al TDAH sugiere que este trastorno genera alteraciones clínicas en el medio familiar, educativo y social de la persona que lo padece. Este significado tiene relación con investigaciones previas, donde se afirma que los niños con TDAH presentan problemática en su hogar y en la escuela (Arango, Mejía, Cardona, \& Cornejo, 2008), o que los adultos con el trastorno presentan dificultades en su desempeño laboral o en su vida afectiva (Barkley \& Fischer, 2011), ya que uno de los componentes que permiten determinar el éxito socioemocional de un adulto, en los diferentes contextos en los que se desenvuelve, más allá de mencionar únicamente el laboral, es prestar una adecuada atención en las diversas actividades que realiza (Mikulic, Crespi, \& Radusky, 2015).

El siguiente significado que emergió en la investigación fue el beneficio que el fármaco puede brindar a la persona que padece TDAH, donde se ha descrito que los niños con el cuadro pueden mejorar su comportamiento luego de recibir este tipo de intervención. Esta categoría tiene relación con investigaciones previas que han reportado que el tratamiento farmacológico de infantes con TDAH mejora en su rendimiento académico (Sandoval, Acosta, Crovetto, \& León, 2009) y funcionamiento cognitivo (Fernández-Jaén, Fernández-Mayoralas, Calleja-Pérez, Moreno-Acero, \& MuñozJareño, 2008).

A pesar de que en un gran porcentaje de medios se ha descrito la bondad del tratamiento farmacológico, se identificó una categoría en la cual se describe que existen efectos no deseados en los niños que lo reciben, lo cual tiene sentido con lo afirmado por Taylor (2014), quien indica que todavía existe controversia en el uso del medicamento como principal estrategia para el tratamiento del TDAH. Algunos ejemplos en favor de dicha aseveración son los reportes de Catalá-López, Ridao, Sanfélix y Preiró (2013) o Valverde e Inchauspe (2014) quienes sugieren que el medicamento en el TDAH no es totalmente efectivo y existen evidencias que describen que quienes reciben este tipo de intervención pueden pre- 
sentar efectos no deseados como insomnio, cefaleas, sintomatología psicótica, trastornos afectivos, entre otros.

En la categoría que engloba las causas del TDAH se refleja el actual conocimiento de este trastorno y se señala que actualmente no se ha llegado a un consenso definitivo en el cual se determinen las causas del TDAH, sino acercamientos desde diversas ópticas que se encuentran aportando en la línea de investigación de este trastorno, que aún se presenta en desarrollo (Ramos \& Pérez-Salas, 2015). En tal sentido, en la presente investigación se hallaron factores biológicos como sociales en la etiología del TDAH, lo cual es concordante con la conceptualización de ser un trastorno multicausal que ha sido descrito en la literatura académica sobre este trastorno (APA, 2015).

En cuanto a la prevalencia del TDAH descrita en los diarios, se afirma correctamente que el TDAH es el trastorno más común en la atención en salud mental infantil. Sin embargo, en el rango de prevalencia se expone que entre el $9.5 \%$ y el $30 \%$ de la población presentaría este trastorno, lo cual es distante de lo sugerido en investigaciones recientes sobre el trastorno en donde se ha descrito que la prevalencia del TDAH se encontraría entre el $6.7 \%$ y el 7.8\% (Ramos, Bolaños, \& Ramos, 2015; Thomas, Sanders, Doust, Beller, \& Glasziou, 2015).

El aporte de esta investigación en la línea de investigación del TDAH, en especial para el contexto ecuatoriano, se proyecta en el sentido de la descripción realizada sobre la construcción que se realiza de este trastorno desde una tarima que capacita y forma los saberes que los individuos poseen sobre los determinados fenómenos que existen en la realidad. De manera que, lo indicado en los reportajes de Ecuador influye en gran medida en los imaginarios que edifican los seres humanos y, en el caso del presente artículo, sobre el TDAH.

En tal sentido, se observa que el contenido que se encontró en los reportajes periodísticos tienen sentido con descripciones teóricas clásicas. Ahora bien, en la actualidad, como se ha dicho anteriormente, en el estudio del TDAH no está establecida la última palabra, ya que es un tema de las ciencias del comportamiento humano que todavía se encuentra en discusión y construcción teórica, por lo que, con la descripción realizada en esta investigación se pretende aportar a un mayor entendimiento del trastorno.

Una limitación del presente estudio que se debe considerar es el aspecto subjetivo del investigador, que si bien se observa que con el análisis del coeficiente Kappa existe una elevada concordancia con la evaluación interjueces, como en todo diseño de investigación cualitativo, dicho factor se convierte en una influencia en el análisis de los datos, a pesar de que en todo momento de la investigación se mantuvo la mayor objetividad en el análisis de la información; sin embargo, es una realidad a la cual no se puede escapar en este tipo de investigaciones.

Otra limitación es que para el análisis se tomaron en consideración únicamente medios de comunicación escrita y no canales de televisión, en donde se hubiera accedido a información más completa sobre el fenómeno de estudio. Sin embargo, esta situación invita a continuar investigando, por ejemplo, como línea de investigación futura, en la que se proyecten estudios para analizar las diferencias entre los hallazgos encontrados en el presente estudio, versus los significados construidos por los canales de televisión.

Finalmente, en cuanto a la generalización de los resultados se debe ser prudente, puesto que la interpretación del TDAH descrita en la presente investigación responde a un contexto determinado, que es el ecuatoriano. No obstante, esta situación es una motivación para realizar investigaciones futuras, en las que se analicen los significados que se construyen en torno a este trastorno en otros contextos como Latinoamérica y en el resto de continentes. 
Tabla 1

Descripción en porcentajes de frecuencias de los reportajes sobre el TDAH publicados en los diarios de Ecuador entre 2007 y 2015

\begin{tabular}{|c|c|c|}
\hline Categoría & Diario & $\%$ \\
\hline \multirow{3}{*}{ El TDAH produce alteraciones familiares, educativas y sociales } & EU & $67 \%$ \\
\hline & EC & $22 \%$ \\
\hline & LH & $11 \%$ \\
\hline \multirow[t]{3}{*}{ El medicamento que lucha contra el TDAH } & EU & $57 \%$ \\
\hline & EC & $14 \%$ \\
\hline & LH & $29 \%$ \\
\hline \multirow[t]{4}{*}{ Concepción del TDAH } & EU & $34 \%$ \\
\hline & EC & $16 \%$ \\
\hline & LH & $34 \%$ \\
\hline & EM & $16 \%$ \\
\hline \multirow[t]{4}{*}{ El TDAH es producto de daño cerebral } & EU & $20 \%$ \\
\hline & EC & $20 \%$ \\
\hline & ED & $40 \%$ \\
\hline & $\mathrm{LH}$ & $20 \%$ \\
\hline \multirow[t]{2}{*}{ Efectos no deseados del fármaco } & EU & $80 \%$ \\
\hline & $\mathrm{LH}$ & $20 \%$ \\
\hline \multirow[t]{3}{*}{ El TDAH es el trastorno más frecuente } & EU & $50 \%$ \\
\hline & EC & $25 \%$ \\
\hline & $\mathrm{LH}$ & $25 \%$ \\
\hline \multirow{2}{*}{ Los dispositivos electrónicos causan TDAH } & EU & $50 \%$ \\
\hline & EC & $50 \%$ \\
\hline \multirow[t]{2}{*}{ La importancia del diagnóstico en el TDAH } & EC & $75 \%$ \\
\hline & $\mathrm{LH}$ & $25 \%$ \\
\hline \multirow[t]{3}{*}{ Factores de riesgo que generan TDAH } & ET & $25 \%$ \\
\hline & EC & $50 \%$ \\
\hline & $\mathrm{LH}$ & $25 \%$ \\
\hline \multirow[t]{4}{*}{ Factores protectores del TDAH } & EU & $25 \%$ \\
\hline & ET & $25 \%$ \\
\hline & $\mathrm{EC}$ & $25 \%$ \\
\hline & ED & $25 \%$ \\
\hline \multirow{3}{*}{$\begin{array}{l}\text { Los medicamentos para el TDAH no aumentan el riesgo de } \\
\text { muerte }\end{array}$} & EU & $50 \%$ \\
\hline & EC & $25 \%$ \\
\hline & ET & $25 \%$ \\
\hline
\end{tabular}

Nota:

EC (El Comercio)

EU (El Universo)

ET (El Telégrafo)

LH (La Hora)

EM (El Mercurio)

ED (El Diario)

UN (Últimas Noticias) 


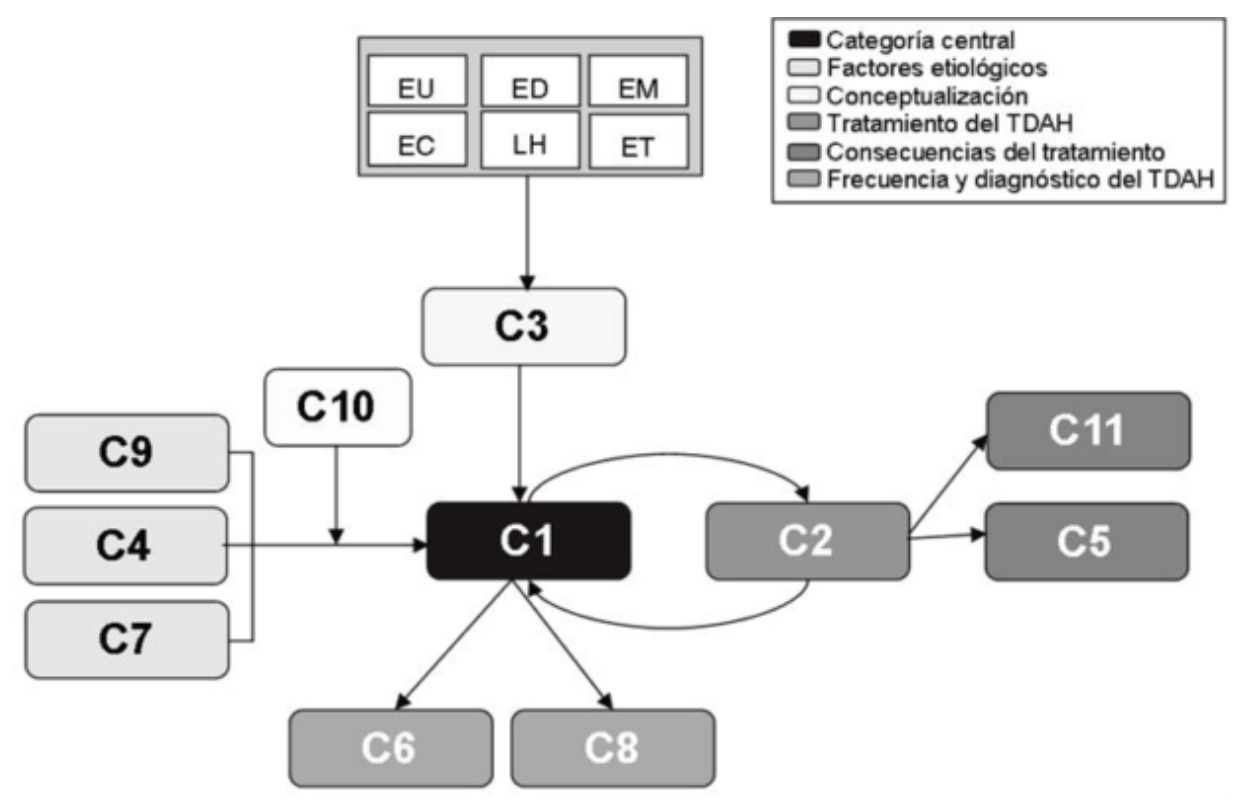

Clave: C1 TDAH produce alteraciones familiares, educativas y sociales, C2 El medicamento que lucha contra el TDAH, C3 concepto del TDAH, C4 TDAH es producto de daño cerebral, C5 efectos no deseados del fármaco, C6 TDAH es el trastorno más frecuente, $\mathrm{C} 7$ dispositivos electrónicos causan TDAH, C8 importancia del diagnóstico en el TDAH, C9 factores de riesgo que generan TDAH, C10 factores protectores en el TDAH, C11 medicamento para el TDAH no aumenta riesgo de muerte.

Figura 1. Paradigma codificado de la teoría emergente del TDAH construido por los medios de comunicación escrita de Ecuador. 


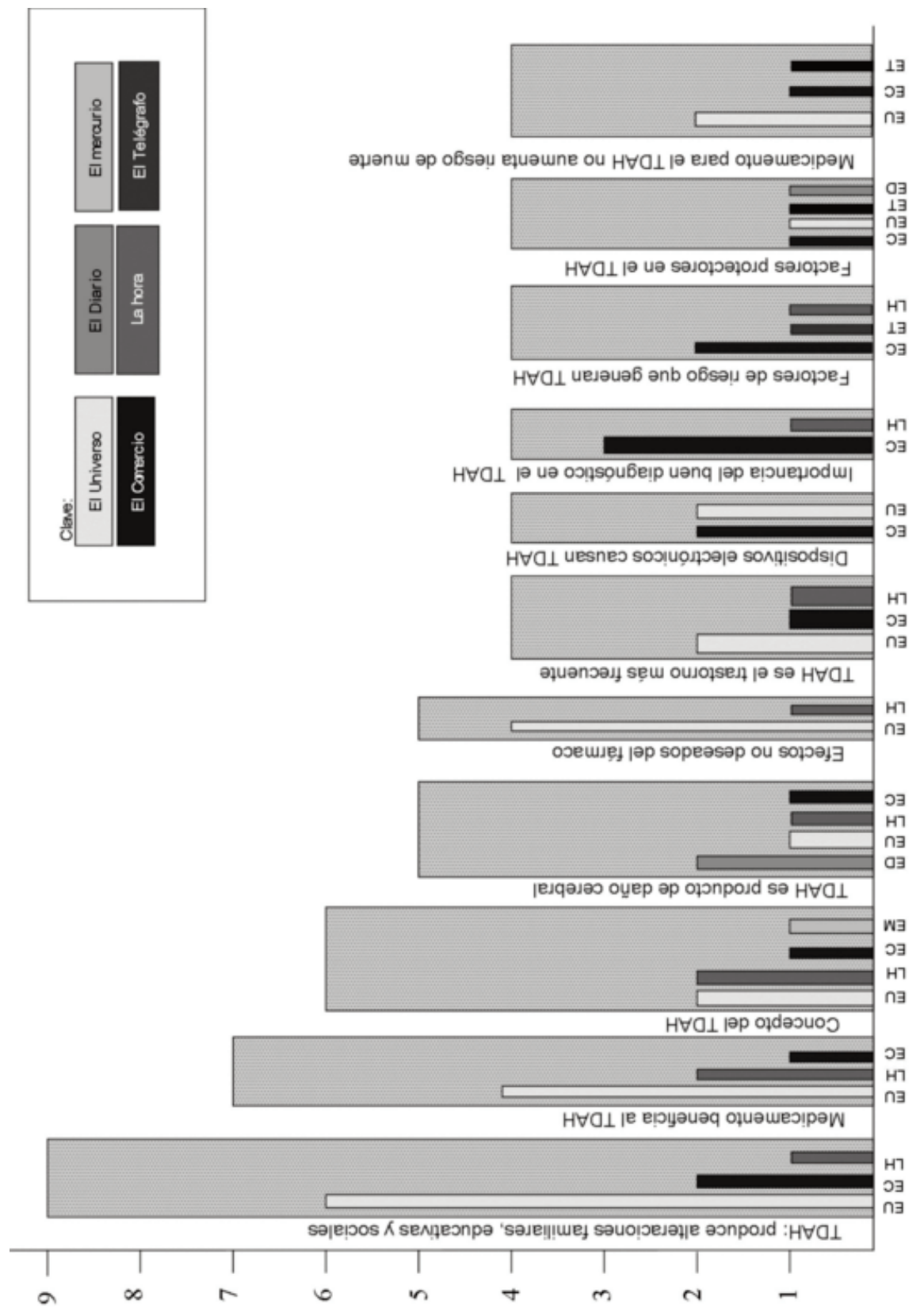

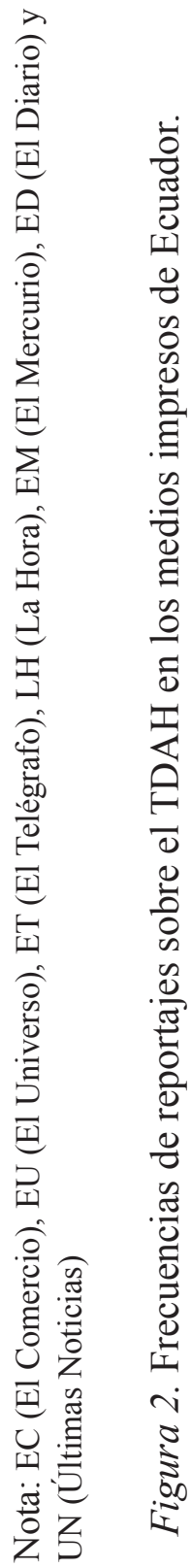




\section{Referencias bibliográficas}

American Psychiatric Association. (2015). Guía de Consulta de los Criterios Diagnósticos del DSM-5. Washington, DC: American Psychiatric Association.

American Psychiatric Association. (2013). Diagnostic and Statistical Manual of Mental Disorders, Fifth Edition (DSM-5). Arlington, VA: American Psychiatric Association.

Anderson, P., \& Reidy, N. (2012). Assessing Executive Function in Preschoolers. Neuropsychology Review, 22, 345-360. http://dx. doi.org/10.1002/mrdd.20075

Arango, L., Mejía, M., Cardona, J., \& Cornejo, J. (2008). Características Clínicas, Neuropsicológicas y Sociodemográficas de Niños Varones con Déficit de Atención/Hiperactividad de Tipo Inatento en Medellín, Antioquia, Colombia 2004- 2005. Iatreia, 21, 375-385.

Barkley, R., \& Fischer, M. (2011). Predicting impairment in Major Life Activities and Occupational Functioning Hyperactive Children as Adults: Self-Reported Executive Function (EF) Deficits Versus EF Tests. Developmental Neuropsychology, 36(2), 137-161. http://dx.doi.org/10.1080/875656 41.2010.549877

Benjamin, P., Peter, M., \& Lynne, R. (2017). Internet Gaming Disorder Explains Unique Variance in Psychological Distress and Disability After Controlling for Comorbid Depression, OCD, ADHD, and Anxiety. Cyberpsychology, Behavior and Social Networking, 20(2), 126-132. http://dx.doi. org/10.1089/cyber.2016.0304.

Biederman, J., Heiligenstein, J., Faries, D., Galil, N., Dittman, R., Emslie, G., \& Schuh, K. (2002). Efficacy of Atomoxetine versus Placebo in school-age girls with AttentionDeficit / Hyperactivity Disorder. Pediatrics, 110(6), e75. http://dx.doi.org/10.1542/peds $.110 .6 . \mathrm{e} 75$

Catalá-López, F., Ridao, M., Sanfélix, G., \& Preiró, S. (2013). Cost-effectiveness of pharmacological treatment of attention deficit hyperactivity disorder in children and adolescents: Qualitative synthesis of scientific evidence. Revista de Psiquiatría y Salud Mental, 6(4), 168-177. http://dx.doi. org/10.1016/j.rpsm.2012.12.002

Cova, F. (2004). La psicopatología evolutiva y los factores de riesgo y protección: el desarrollo de una mirada procesual. Revista de Psicología de la Universidad de Chile, 13(1), 93-101. http://dx.doi.org/10.5354/ 0719-0581.2004.17489

De la Barra, F., Vicente, B., Saldivia, S., \& Melipillan, R. (2013). Epidemiology of ADHD in Chilean children and adolescents. ADHD Attention Deficit and Hyperactivity Disorders, 5(1), 1-8. http://dx.doi.org/10.10 07/s12402-012-0090-6

E1 Comercio (2010a). Los familiares de Lindsay Lohan la visitan en prisión. Recuperado el 17 de febrero de 2017 de http://www.elcomercio.com/tendencias/entretenimiento/familiares-lindsay-lohan-visitan-prision.html.

El Comercio (2010b). ¿Por qué cada vez más niños/as hacen terapia? Recuperado el 18 de febrero de 2017 de http://www.elcomercio.com/actualidad/mundo/vez-mas-ninosterapia.html.

El Comercio (2010c). El varón tiene más riesgo de sufrir accidentes. Recuperado el 19 de febrero de 2017 de http://www.elcomercio. com/tendencias/varon-mas-riesgo-sufrir-accidentes.html.

El Comercio (2011a). Descartan riesgo cardíaco de fármacos para el déficit atencional. Ecuador. Recuperado el 17 de febrero de 2017 de http://www.elcomercio.com/tendencias/salud/descartan-riesgo-cardiaco-farmacos-deficit.html.

El Comercio (2011b). Asocian humo de segunda mano con problemas de conducta en niños/as. Recuperado el 20 de febrero de 2017 de http://www.elcomercio.com/tendencias/salud/asocian-humo-segunda-manoproblemas.html. 
El Comercio (2012a). Expertos podrían predecir el riesgo psiquiátrico por computadora. Recuperado el 16 de febrero de 2017 de http://www.elcomercio.com/tendencias/salu $\mathrm{d} /$ expertos-predecir-riesgo-psiquiatricocomputadora.html.

El Comercio (2012b). Exposición de mujeres embarazadas a radiaciones de celulares puede provocar problemas en el comportamiento infantil. Recuperado el 22 de febrero de 2017 de http://www.elcomercio.com/tendencias/salud/exposicion-mujeres-embarazadas- radiaciones-celulares.html.

El Comercio (2012c). Las tabletas electrónicas y los niño/as un delicado equilibrio. Recuperado el 25 de febrero de 2017 de http:// www.elcomercio.com/tendencias/ciencia/tabletas-electronicas-y-ninos-delicado.html.

El Comercio (2012d). Fármacos VIH no están ligados a problemas psiquiátricos en niños/as. Recuperado el 25 de febrero de 2017 de http://www.elcomercio.com/tendencias/ salud/farmacos-vih-no-ligados-problemas.html.

El Comercio (2013a). Enfermedades mentales comparten factores de riesgo genéticos, según estudio. Recuperado el 17 de febrero de 2017 de http://www.elcomercio.com/tendencias/salud/enfermedades- mentales-comparten-factores-de.html.

El Comercio (2013b). Los aditivos en exceso no son buenos. Recuperado el 18 de febrero de $2017 \mathrm{de} \mathrm{http://www.elcomercio.com/ten-}$ dencias/salud/aditivos-exceso-no-son-buenos.html.

El Comercio (2015a). Las personas con déficit de atención sufren mayor riesgo de muerte prematura. Recuperado el 18 de febrero de $2017 \mathrm{de}$ http://www.elcomercio.com/tendencias/personas-deficitdeatencion-muerteprematura- estudio-riesgos.html.

El Comercio (2015b). El TDAH se supera con un tratamiento adecuado y oportuno en la niñez. Recuperado el 21 de febrero de 2017 de http://www.elcomercio.com/tendencias/ tdah-salud-tratamiento-ninez-trastor nos.html.

El Diario (2013). Amamantar puede reducir el riesgo de hiperactividad infantil. Recuperado el 24 de febrero de 2017 de http:// www.eldiario.ec/noticias-manabi-ecuador/280009-amamantar-puede-reducir-elriesgo-de-hiperactividad-infantil-segun-estu dio/.

El Diario (2014). Trastorno de atención es investigado. Recuperado el 25 de febrero de 2017 de http://www.eldiario.ec/noticiasmanabi-ecuador/338176-trastorno-de-atencion-es-investigado/.

El Mercurio (2011). Fármacos para déficit de atención no tienen riesgo para el corazón. Recuperado el 17 de febrero de 2017 de http:/www.elmercurio.com.ec/312798-farmacos-para-deficit-de-atencion-no-tienenriesgo-para-el-corazon/\#.Vf8wP5f0fT8.

El Mercurio (2013). Déficit de atención e hiperactividad. Recuperado el 19 de febrero de 2017 de http://www.elmercurio.com.ec/ 377917-deficit-de-atencion-e-hiperactividad/\#.Vf8wO5f0fT8.

El Telégrafo (2013a). Dar el seno podría reducir casos de hiperactividad.Recuperado el 21 de febrero de 2017 de http://telegrafo.com. ec/sociedad/item/dar-el-seno-podria-reducir-casos-de- hiperactividad.html.

El Telégrafo (2013b). Estudio asocia el azúcar con desarrollo de la hiperactividad. Recuperado el 25 de febrero de 2017 de http:// www.telegrafo.com.ec/sociedad/item/estudio-asocia-el-azucar-con-desarrollo-de-lahiperactividad.html.

El Telégrafo (2014). Los antidepresivos tendrían relación con la hiperactividad. Recuperado el 25 de febrero de 2017 de http:// telegrafo.com.ec/sociedad/item/los-antidepresivos-tendrian-relacion-con-la-hiperactividad.html.

El Universo (2009). Medicamentos para hiperactivos pueden causar alucinaciones en 
niño/as. Recuperado el 25 de febrero de 2017 de http://www.eluniverso.com/2009/ 01/26/1/1384/E140FF4A879D4D96B5746F 811FC64BFF.html.

El Universo (2010). Niño/as hiperactivos podrían sufrir de depresión en la adolescencia. Recuperado el 20 de febrero de 2017 de http://www.eluniverso.com/2010/10/06/1/13 84/ninos- hiperactivos-podrian-sufrir-depresion-adolescencia-II.html.

El Universo (2011a). Fármacos para déficit de atención no elevan cardiopatías. Recuperado el 17 de febrero de 2017 de http://www.eluniverso.com/2011/11/02/1/1384/farmacosdeficit-atencion-elevan-cardiopatias.html

El Universo (2011b). Fármacos para déficit de atención no tienen riesgo para el corazón. Recuperado el 24 de febrero de 2017 de http://www.eluniverso.com/2011/12/13/1/13 84/farmacos-deficit-atencion-tienen-riesgocorazon.html.

El Universo (2011c). La dificultad para poner atención. Recuperado el 24 de febrero de 2017 de http://www.eluniverso.com/2011/ 09/22/1/1550/dificultad-poner-atencion. html.

El Universo (2012a). Lo que sienten los niño/as hiperactivos. Recuperado el 17 de febrero de 2017 de http://www.eluniverso.com/2012/ 10/28/1/1384/lo-sienten-ninos-hiperactivos.html.

El Universo (2012b). Tabletas electrónicas y niño/as, una delicada unión. Recuperado el 17 de febrero de 2017 de http://www.eluniverso.com/2012/04/03/1/1431/tabletas-electronicas-ninos-delicada-union.html.

El Universo (2012c). Agencia de alimentos y medicamentos alerta por venta de fármaco falso para déficit de atención. Recuperado el 18 de febrero de 2017 de http://www.eluniverso.com/2012/05/30/1/1384/agencia-alimentos-medicamentos-alerta-venta-farma co-falso-deficit-atencion.html.

El Universo (2012d). La radiación del teléfono móvil afecta al cerebro de los fetos de ratón. Recuperado el 19 de febrero de 2018 de http://www.eluniverso.com/2012/03/15/1/13 84/radiacion-telefono-movil-afecta-cerebrofetos-raton-segun-estudio.html.

El Universo (2013a). El azúcar en los niño/as, ¿realmente causa hiperactividad? Recuperado el 20 de febrero de 2017 de http:// www.eluniverso.com/vida-stilo/2013/ 09/28/nota/1508356/azucar-ninos-realmente-causa-hiperactividad.

El Universo (2013b). La hiperactividad se vincula con el autismo. Recuperado el 20 de febrero de 2017 de http://www.eluniverso. com/vida-estilo/2013/05/05/nota/902236/hiperactividad-se- vincula-autismo.

El Universo (2015b). Las razones para tener un gato como mascota. Recuperado el 20 de febrero de 2017 de http://www.eluniverso. com/noticias/2015/04/09/nota/4747011/razones-tener-gato-como-mascota.

Fernández-Jaén, A., Fernández-Mayoralas, D., Calleja-Pérez, B., Moreno-Acero, N., \& Muñoz- Jareño, N. (2008). Efectos del metilfenidato en los procesos cognitivo- atencionales. Uso de los test de ejecución continuada. Revista de Neurología, 46(1), S47S49.

Graham, S. (2017). Attention-deficit Hyperactivity Disorder (ADHD), Learning Disabilities (LD), and executive functioning: Recommendations for future research. Contemporary Educational Psychology, 50, 97-101. http://dx.doi.org/10.1016/j.ced psy ch.2017.01.001.

Hernández, R., Fernández, C., \& Baptista, P. (2010). Metodología de la Investigación. México D.F.: Mc Graw-Hill/Interamericana Editores.

Hueso, A., \& Cascant, M. (2012). Metodología y técnicas cuantitativas de investigación. Cuadernos docentes en procesos de desarrollo. Valencia: Editorial Universitat Politécnica.

IBM. (2011). SPSS Statistics. IBM Corp.

La Hora (2007). Problemas mentales tienen tratamiento. Recuperado el 20 de febrero de 
2017 de http://www.lahora.com.ec/index. $\mathrm{php} /$ noticias/show/610666/-1/Problemas mentales_tienen_tratamiento.html\#.Vf75b5f 0fT8.

La Hora (2012a). Evidencia científica. Recuperado el 17 de febrero de 2017 de http://www.lahora.com.ec/index.php/noticias/show/1101341653/-1/home/go Regional/Loja\#.Vf75Y5f0fT8.

La Hora (2012b). Ronquidos pueden causar problemas. Recuperado el 20 de febrero de 2017 de http://www.lahora.com.ec/index. php/noticias/show/1101297181/1/Ronquidos_pueden_causar_problemas.ht $\mathrm{ml} \#$.Vf75a5f0fT8.

La Hora (2012c). Hiperactividad infantil: información simple sobre un tema complejo. Recuperado el 22 de febrero de 2017 de http://www.lahora.com.ec/index.php/noticias/show/1101350657/-1/home/goRegio nal/Quito\#.Vf75X5f0fT8.

La Hora (2015). Desfogue. Recuperado el 18 de febrero de 2017 de http://www.lahora. com.ec/index.php/movil/noticia/1101 $354829 / \mathrm{Ni} \% \mathrm{C} 3 \% \mathrm{~B} 1$ os_inquie tos,_\%C2\%B Ftienen_un_problema\%3F.html.

Mikulic, I., Crespi, M., \& Radusky, P. (2015). Construcción y validación del inventario de competencias socioemocionales para adultos (ICSE). Interdisciplinaria, 32(2), 307330.

Miranda, A., \& Presentación, M. (2000). Efficacy of cognitive-behavioral therapy in the treatment of children with ADHD, with and without aggressiveness. Psychology in the Schools, 37(2), 169-182. http://dx.doi. org/10.1002/(SICI)1520-6807(200003) 37:2<169::AID- PITS8>3.0.CO;2-8

Monteoliva, J., Ison, M., \& Pattini, A. (2014). Evaluación del desempeño atencional en el niño: eficacia, eficiencia y rendimiento. Interdisciplinaria, 31(2), 213-225.

Naaijen, J., Bralten, J., Poelmans, G., ImageConsortium, Glennon, J., Franke, B., \&
Buitelaar, J. (2017). Glutamatergic and GABAergic gene sets in attention- deficit/hyperactivity disorder: association to overlapping traits in ADHD and autism. Translational Psychiatry, 7, 1-7. http://dx. doi.org/10.1038/tp.2016.273

Nigg, J. (2012). Future directions in ADHD etiology research. Journal of Clinical Child \& Adolescent Psychology, 41(4), 524-533. http://dx.doi.org/10.1080/15374416.2012.68 6870

Ramos, C. (2015). Los paradigmas de la investigación científica. Avances en Psicología, 23(1), 9-17.

Ramos, C., Bolaños, M., \& Ramos, D. (2015). Prevalencia del TDAH en estudiantes ecuatorianos. Revista Científica y Tecnológica, 3(1), 13-19.

Ramos, C., \& Pérez-Salas, C. (2015). Relación entre el modelo hibrido de las funciones ejecutivas y el trastorno por déficit de atención con hiperactividad. Psicología desde el Caribe, 32(2), 299-314. http://dx.doi.org/ 10.14482/psdc.32.2.5986

Raposo-Rivas, M., \& Salgado-Rodríguez, A. (2015). Estudio sobre la intervención con Software educativo en un caso de TDAH. Revista Nacional e Internacional de Educación Inclusiva, 8(2), 121-138.

Roca, P., Presentación-Herrero, M., MirandaCasas, A., Mulas, F., \& Ortiz- Sánchez, P. (2014). El componente P300 como correlato neurofisiológico de la memoria de trabajo conductual en adolescentes con trastorno por déficit de atención con hiperactividad. Revista de Neurología, 58(1), S51-S56.

Sandoval, A., Acosta, N., Crovetto, F., \& León, M. (2009). Enfrentamiento terapéutico del trastorno por déficit atencional en una población infantil escolar perteneciente a la Región Metropolitana de Chile. Revista Chilena de Neuro-Psiquiatría, 47(1), 34-42. http://dx.doi.org/10.4067/S0717-92272009 000100005 
Strauss, A., \& Corbin, J. (2002). Bases de la investigación cualitativa. Técnicas y procedimientos para desarrollar la teoría fundamentada. Antioquia, Colombia: Editorial Universidad de Antioquia.

Taylor, E. (2014). Uses and misuses of treatments for ADHD. The second Birgit Olsson lecture. Nordic Journal of Psychiatry, 68, 236-242. http://dx.doi.org/10.3109/080394 88.2013.804118

Thomas, R., Sanders, S., Doust, J., Beller, E., $\&$ Glasziou, P. (2015). Prevalence of attention- deficit/hyperactivity disorder: a systematic review and a meta-analysis. Pediatrics, 135(4), 994-1001. http://dx.doi.org/ 10.1542/peds.2014-3482

Últimas Noticias. (2011). ¿Vago? no, hiperactivo. Recuperado el 21 de febrero de 2017 de http://www.ultimasnoticias.ec/noticias/ 3922-ivago-no-hiperactivo.html.

Últimas Noticias. (2013). Un gol con la imaginación. Recuperado el 22 de febrero de 2017 de: http://www.ultimasnoticias.ec/noticias/ 12962-un-gol-con-la-imaginacion.html.
Valverde, M., \& Inchauspe, J. (2014). Alcance y limitaciones del tratamiento farmacológico del Trastorno por Déficit de Atención e Hiperactividad en niño/as y adolescentes y Guías de Práctica Clínica. Una revisión bibliográfica. Revista de la Asociación Española de Neuropsiquiatría, 27-34. http://dx. doi.org/10.4321/S0211-573520140001000 04

Vélez Van Meerbeke, A., Zamora, I., Guzmán, B., López, C., \& Talero-Gutierrez, C. (2013). Evaluación de la función ejecutiva en una población escolar con síntomas de déficit de atención e hiperactividad. Neurología, 28(6), 348-355. http://dx.doi.org/10.1016/ j.nrl.2012.06.011

Winther, E., Egeland, J., Norman, P., Tore, K., \& Øie, M. (2014). Few differences in hot and cold executive functions in children and adolescents with combined and inattentive subtypes of ADHD. Child Neuropsychology: A Journal on Normal and Abnormal Development in Childhood and Adolescence, 20(2), 162-181. http://dx.doi.org/ 10. 1080/09297049.2012.753998

Recibido: 11 de noviembre de 2016 Aceptado: 21 de junio de 2018 